\title{
A MULHER E A PESCA: UM OLHAR SOBRE A PESQUISA E A ATUAÇÃO FEMININA PESQUEIRA NO BRASIL
}

\author{
WOMEN AND FISHERIES: A LOOK AT RESEARCH AND FEMALE FISHING \\ ACTIVITIES IN BRAZIL
}

Priscila Fabiana Macedo LOPES ${ }^{1,2 *}$; Carolina Tavares de FREITAS ${ }^{3}$; Alpina BEGOSSI ${ }^{2,4}$

1 - Fishing ecology, management, and economics group. Departamento de Ecologia, Universidade Federal do Rio Grande do Norte (UFRN), Campus Universitário - Lagoa Nova, 59078-900 - Natal, RN, Brasil; 2 - Fisheries and Food Institute - FIFO, Santos, SP, Brasil; 3 - Divisão de Sensoriamento Remoto, Coordenação de Observação da Terra, Instituto Nacional de Pesquisas Espaciais, São José dos Campos, SP, Brasil; 4 - Núcleo de Estudos e Pesquisas em Alimentação - NEPA, Universidade Estadual de Campinas (UNICAMP), Campinas, SP, Brasil. *priscila@ cb.ufrn.br;

\section{RESUMO}

Submitted: 04/06/2020; Accepted: 17/07/2020

As mulheres pescadoras lidam historicamente com a falta de reconhecimento de suas atividades, o que tem implicações socioeconômicas (e.g., falta de remuneração por seu trabalho) e de saúde (e.g.: falta de reconhecimento de suas doenças trabalhistas). Através de uma revisão da literatura, aqui avaliamos eventuais mudanças neste cenário para as pescadoras brasileiras, incluindo o seu reconhecimento na literatura científica. Encontramos que, nas últimas décadas, as pescadoras brasileiras conseguiram melhorias: muitas, por exemplo, gerenciam diferentes associações de pescadores em todo o país, incluindo algumas historicamente dominadas por homens, como as colônias de pesca. A academia também reflete esta tendência ao mostrar crescente interesse no entendimento do papel das mulheres na pesca brasileira. No entanto, as capturas femininas ainda tendem a não ser registradas, embora os peixes e mariscos que coletam tenham um papel central na subsistência, segurança e soberania alimentar de suas famílias e comunidades. Por outro lado, à medida que o reconhecimento feminino progride lentamente, em alguns casos as mulheres começam a ter seu trabalho valorizado economicamente para além das esferas de processamento e venda de peixes. Alguns exemplos mostram que trabalhos de pesca tradicionalmente vistos como uma simples "ajuda ao parceiro" começam a ser economicamente remunerados, o que tende a se traduzir em melhor uso desta renda no bem-estar familiar. Estas conquistas positivas ajudam a revelar barreiras mais sutis, como a constante luta das mulheres para serem reconhecidas como trabalhadoras da pesca quando seu trabalho não está diretamente no setor extrativo (mas voltado, por exemplo, à fabricação de equipamentos ou ao processamento e comércio de peixes). Mesmo quando diretamente envolvidas no setor extrativo, as pescadoras ainda lutam para ter o reconhecimento de suas doenças ocupacionais, sem o qual seu acesso aos direitos trabalhistas é limitado. Assim, há muito a ser feito para que o trabalho da pesca feminina saia da invisibilidade, mas os primeiros passos já foram dados, cabendo à sociedade como um todo propulsionar esse processo.

Palavras-chave: Invisibilidade pesqueira, Gestão pesqueira feminina, Comanejo pesqueiro, Pescadora artesanal

\begin{abstract}
Fisherwomen have historically dealt with the lack of recognition of their activities, with implications on socioeconomic aspects (e.g., lack of remuneration for their work) and health problems (e.g., lack of recognition of their labor diseases). Through a literature review, here we evaluated eventual changes in this scenario for Brazilian fisherwomen, including their recognition in the scientific literature. We found that, in recent decades, Brazilian fisherwomen have achieved some improvements: many, for example, manage different fishing associations across the country, including some historically dominated by men, such as fishing colonies. The academy also reflects this trend by showing growing interest in understanding the role of women in Brazilian fishing. However, female catches still tend not to be recorded, although the fish and shellfish they collect play a central role in the subsistence, security and food sovereignty of their families and communities. On the other hand, as female recognition progresses slowly, in some cases women begin to have their work economically valued beyond fish processing and selling. Some examples show that fishing jobs traditionally seen as simply "helping the partner" begin to be economically remunerated, which tends to translate into better use of this income for family wellbeing. These positive achievements help reveal more subtle barriers, such as the constant struggle of women to be recognized as fisherworkers when their work is not in the harvest sector (but focused, for example, on gear manufacture or fish processing and trade). Even when directly involved in harvesting fish, fisherwomen still struggle to gain recognition for their occupational diseases, without which their access to labor rights is limited. Thus, there is much to be done to make the work of female fishing out of invisibility, but the first steps have already been taken, and it is up to society as a whole to boost this process.
\end{abstract}

Keywords: Fishing invisibility, Female fisheries management, Fisheries co-management, Artisanal fisherwoman. 


\section{INTRODUÇÃO}

Naquele que é talvez um dos livros mais famosos de Ernest Hemingway, "O Velho e o Mar", o experiente pescador Santiago, após uma longa maré de má sorte, finalmente fisga (ou é fisgado por) um grande Marlim, que o arrasta para alto mar por dias. Após uma batalha épica, Santiago retorna à terra, mas seu peixe é devorado no caminho por tubarões. No entanto, perder todo o peixe não é tão relevante, pois Santiago é recebido com respeito e admiração pelos demais pescadores, todos homens. Para além da literatura, há certa romantização do pescador, o homem sofrido, mas viril, que enfrenta o mar, nem sempre ganha a batalha (ou o peixe de cada dia), embora frequentemente ganhe o respeito de seus companheiros à medida que enfrenta perigos (BÉNÉ; TEWFIK, 2001; HAWKES; BIRD, 2002; SZYMKOWIAK, 2020). Ainda que parte desta descrição seja justa e que de fato a pesca seja uma atividade dura e muito pouco reconhecida, especialmente a de pequena escala (i.e., com baixa autonomia e investimento tecnológico, e baseada na organização familiar) (BÉNÉ; MACFADYEN; ALLISON, 2007; DAMÁSIO; PENINNO; LOPES, 2020), este quadro é incompleto. Há mais a ser dito por trás do pouco reconhecimento socioeconômico do pescador e da glorificação dos riscos da atividade (ZHAO et al., 2013). Há de ser contado que a pesca não é praticada só por homens, que o peixe não é comercializado só por homens, que toda a sua cadeia produtiva não é formada só por homens, que a segurança alimentar das comunidades pesqueiras não é garantida só pelos homens. Ou melhor, há de ser contado que as mulheres também pescam, as mulheres também vendem o peixe, por vezes até mais que os homens, as mulheres fazem parte de toda a cadeia produtiva da pesca, da rede ao prato e, não raro, as mulheres garantem a segurança alimentar da família por meio da pesca até mais que os homens (HARPER et al., 2013, 2020; KLEIBER; HARRIS; VINCENT, 2014a).

Por que então as mulheres ainda seguem sendo invisíveis na atividade? Por que esta invisibilidade se traduz inclusive na academia, onde ainda são proporcionalmente tão escassos os estudos sobre o papel feminino na pesca? A academia refletiria de fato uma menor relevância feminina na pesca? Ou a própria academia adota o mesmo olhar enviesado da sociedade, ignorando que quase sempre a pesca, especialmente a de pequena escala, só é possível devido também à participação das mulheres? São muitas perguntas que se desenham, muitas das quais ficarão sem uma discussão aprofundada aqui, no contexto brasileiro, por ausência de dados, mas que vêm sendo finalmente exploradas ao redor do mundo. No Brasil, os estudos de gênero na pesca ainda engatinham, mas apontam para uma realidade não tão dissonante dos demais países. Nesta revisão, objetivamos analisar os diferentes papeis femininos na cadeia produtiva da pesca e na manutenção da segurança alimentar das famílias que dependem direta ou indiretamente da atividade no Brasil, e as consequências da inclusão feminina na gestão e na tomada de decisão pesqueira.

\section{Um olhar sobre a pesquisa do papel feminino na pesca}

A questão de gênero na pesca surge na literatura acadêmica especializada primeiramente em livros. Dentre estes, destacam-se alguns estudos nas décadas de 1960 e 1970, que já enfatizavam o papel das mulheres nas comunidades pesqueiras, especialmente no processamento e comércio de pescado (CHRISTENSEN, 1977; FIRTH, 1966). Alguns deles, como os realizados na Malásia e em Gana, mostram que o empoderamento feminino na pesca se dava através da aquisição de material pesqueiro ou controle parcial ou total do comércio do peixe (CHRISTENSEN, 1977; FIRTH, 1966) (Quadro 1).

Apenas mais tarde, o interesse parece começar a se manifestar de maneira mais evidente nos periódicos científicos. Em uma busca na base de dados Scopus, limitada às palavras "fishery" or "fisheries" or "fishing" and "women", cujos resultados foram refinados para aqueles que continham a palavra "gender" e depois limpos para exclusão de artigos não relacionados (por exemplo, aqueles que investigavam a propagação de HIV em vilas pesqueiras africanas ou contaminação por mercúrio), obtivemos 262 artigos publicados até o fim de março de 2020. Dentre estes artigos encontrados, o primeiro deles data de 1985, o qual já traz muitas das questões que ainda hoje permeiam o tema, como a necessidade de ver a mulher como parte integrante da pesca e o reconhecimento de que o homem frequentemente depende dela para lidar com o peixe depois de capturado (THOMPSON, 1985). Nestes estudos já há um questionamento sobre o 'male bias' (viés masculino) da abordagem pesqueira, sugerindo que a academia estaria acordando para isto (JENTOFT, 2005). Além de comunidades pesqueiras na África (e.g. Togo, Gana), onde a participação das mulheres já era mais conhecida, as mariscadoras ou marisqueiras começam a ganhar destaque, especialmente as atuantes mariscadoras da Galícia, Espanha (JENTOFT, 2005). 
Quadro 1. Alguns dos primeiros registros na literatura internacional do papel feminino na pesca.

a) Os Fanti de Gana e os pescadores da Malásia (CHRISTENSEN, 1977)

Os Fanti fazem parte do grupo Akan, do sudeste de Gana. Ao longo do tempo, os pescadores Fanti substituíram suas canoas a remo por canoas a motor, o que lhes permitiria ter tripulações e redes maiores. No entanto, na ausência de apoio governamental, poucos tinham capital suficiente para equipar suas novas embarcações ou contratar pescadores adicionais. Este capital foi então suprido através de financiamentos providos especialmente pelas mulheres, as chamadas market women, que detinham algum poder econômico por já controlarem o processamento e o comércio local de pescado. $\mathrm{O}$ estabelecimento destes financiamentos, inexistentes até então, subsidiaram não apenas equipamentos, mas também momentos de escassez da pesca. Essa foi uma mudança socioeconômica importante, que levou inclusive ao estabelecimento de relações de trabalho entre mulheres e pescadores homens. Ainda assim, muitos pescadores relutavam em admitir a sua dependência das mulheres.

b) Os pescadores malaios (FIRTH, 1966)

Calantão é um estado da Malásia onde predominam a pequena agricultura do arroz e a pesca artesanal. Em 1957, o Calantão possuía aproximadamente 6.500 pescadores, ou pessoas ligadas a atividades pesqueiras, correspondendo a $3 \%$ de sua população. Nessa comunidade, as mulheres influenciavam as decisões masculinas, não só no ambiente familiar, mas também como conselheiras ou mesmo liderando as questões relativas a investimentos. A comercialização do peixe dividia-se entre homens e mulheres, a depender do mesmo ser vendido fresco (venda masculina) ou processado - cozido ou curado (venda feminina). Embora este livro não se atente especificamente a questões de gênero, já ilustra o envolvimento feminino na cadeia produtiva do peixe.

Apesar de o interesse acadêmico ter se mantido relativamente incipiente desde meados da década de 1980, atualmente ele parece vir seguindo um crescimento exponencial. Somente entre 2018 e março de 2020, por exemplo, 77 artigos científicos foram publicados sobre o tema (Figura 1; Material suplementar), com destaque para o periódico Maritime Studies, que organizou alguns volumes especiais sobre o assunto e abarcou, neste período, $30 \%$ das publicações sobre a participação feminina na pesca. Os estudos ainda são poucos na América Latina e Caribe, embora haja evidências da atuação da mulher em várias etapas das atividades de pesca, seja na preparação de iscas (Uruguai), coleta e mariscagem (Brasil), limpeza, evisceração e processamento de pescado (El Salvador) ou no manejo e organização da pesca (vários países do Caribe) (DE OLIVEIRA LEIS et al., 2019). No Brasil especificamente, o primeiro estudo que encontramos em um periódico indexado data de 2007 (MAGALHÃES et al., 2007). Apesar de considerarmos que outras pesquisas devem estar disponíveis em capítulos de livros e na literatura cinza em períodos anteriores a este e que as palavras-chave utilizadas em nossa busca podem não ter sido exaustivas, parece-nos evidente que este ainda é um campo novo no país e que a participação feminina na pesca precisa ser melhor explorada nas pesquisas científicas.

$\mathrm{O}$ surgimento destes estudos começa a trazer luz à ação feminina no universo pesqueiro e a apontar os motivos pelos quais o papel da mulher vem sendo tão negligenciado nesse universo. Dentre as principais razões que podem ser atribuídas a esta invisibilidade, muitas estão associadas a questões bioculturais. Mulheres tendem, por exemplo, a concentrar as atividades pesqueiras em ambientes próximos à sua área de residência, como zonas costeiras, estuários e mangues (ARCE-IBARRA; CHARLES, 2008). Tal proximidade normalmente se faz necessária por permitir que elas levem seus filhos consigo e/ou retornem rapidamente para casa a fim de cuidar deles e dos demais afazeres domésticos, responsabilidades atribuídas à mulher em muitas culturas (FRÖCKLIN et al., 2013; ROCHA; PINKERTON, 2015; WEERATUNGE; SNYDER; SZE, 2010). Além disso, esses ambientes são também considerados "mais seguros" que o alto-mar (ARCE-IBARRA; CHARLES, 2008) e frequentemente tidos como mais "adequados" à mulher. No entanto, isto não quer dizer que mulheres também não se arrisquem por águas mais profundas, enquanto homens ficam em casa (BRUMMETT et al., 2010). Tais papéis e a "coragem" atribuída a homens e mulheres variam de acordo com construções socioculturais e o que cada sociedade espera de cada gênero. 


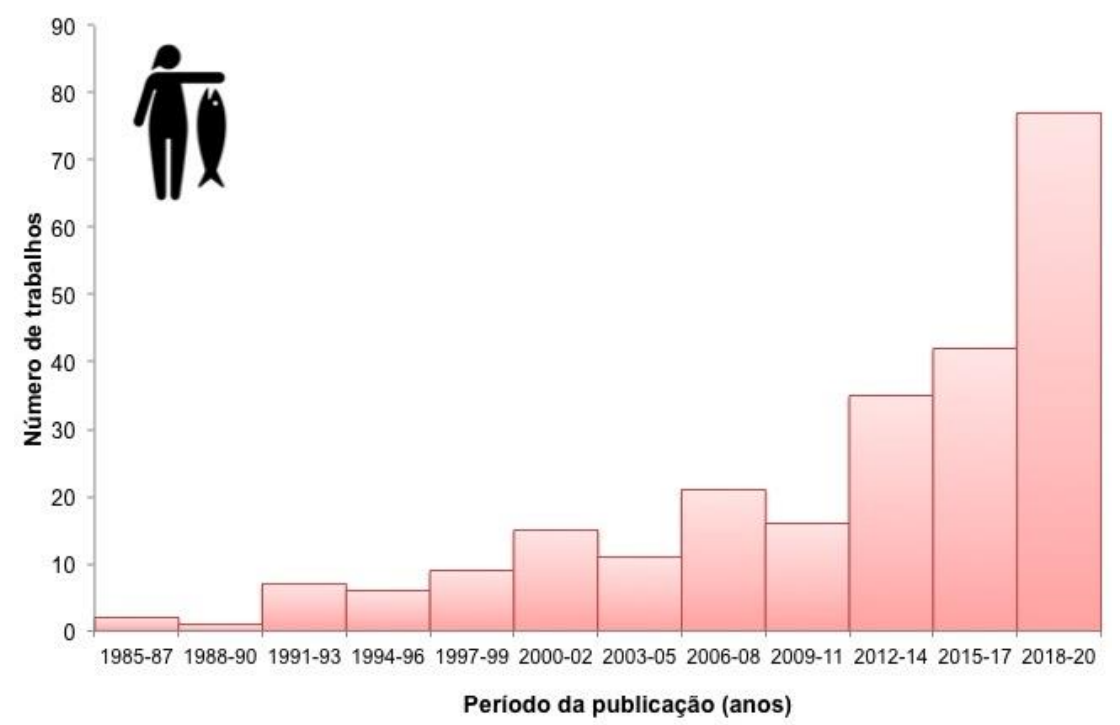

Figura 1: Evolução temporal no número de artigos científicos abordando a participação feminina em atividades de pesca, segundo levantamento feito na Plataforma Scopus. O ano de 2020 computa apenas os trabalhos publicados disponíveis até o dia 31 de março.

Por atuarem em ambientes mais específicos, também é comum que mulheres se especializem na cata de invertebrados ou de pequenos peixes. Isto faz com que muitos, desde os órgãos responsáveis pelas estatísticas pesqueiras até as próprias mulheres, não as classifiquem como pescadoras (OGDEN, 2017). São chamadas de marisqueiras, mariscadoras, gleaners etc., conforme o idioma, mas raramente de pescadoras (BRANCH; KLEIBER, 2017). Reconhecê-las formalmente como pescadoras, independentemente da denominação que optarem, incluindo-as nas estatísticas pesqueiras e nas pesquisas que acompanham desembarques pesqueiros ou que investigam o conhecimento ecológico local, é um primeiro passo essencial. Além disso, é fundamental entender o trabalho pesqueiro não apenas como a atividade de ir ao mar ou ao rio buscar o peixe, mas também o trabalho realizado em terra, do processamento do pescado à manutenção dos apetrechos pesqueiros (KERGOAT, 2009). A partir disto, entenderemos melhor em que consiste a segurança alimentar e o bem-estar das comunidades pesqueiras com e sem a participação feminina (RETIRED; MBEZI, 2010), no que consiste a cadeia produtiva da pesca artesanal com e sem a mulher (WEERATUNGE; SNYDER; SZE, 2010), bem como o que esperar da gestão da pesca quando as mulheres são ou não incluídas (FRÖCKLIN et al., 2013). Talvez descubramos que a invisibilidade feminina nas políticas públicas e nos trabalhos acadêmicos apenas reflete a desigualdade socioeconômica a que as mulheres estão sujeitas em praticamente todas as esferas da sociedade (COFFEY et al., 2020). Por fim, desvendar o papel feminino na pesca talvez implique em desvendar perspectivas inovadoras de extração e gestão de recursos pesqueiros que podem auxiliar na sustentabilidade desta atividade (CALHOUN; CONWAY; RUSSELL, 2016).

\section{A mulher, as estatísticas pesqueiras, o comércio de pescado e a segurança alimentar de comunidades pesqueiras}

A invisibilidade da mulher nas atividades de pesca é notável mesmo em setores que elas dominam, como nas atividades pós-captura. Na Índia, por exemplo, cerca de 75\% da força de trabalho nas atividades alimentares pesqueiras é feminina (JEYANTHI et al., 2015). Além disso, sabe-se que as mulheres possuem importância chave em vários elos da cadeia produtiva pesqueira em todo o mundo (Quadro 2) como: 1) usuárias primárias (quando pescam sozinhas ou financiam operações de pesca), 2) usuárias secundárias (quando obtêm peixes por parentesco ou outros relacionamentos próximos) e 3) usuárias terciárias (quando usam capital para comprar peixe diretamente de pescadores ou comerciantes) (LENTISCO; LEE, 2015).

Apesar da evidente importância das mulheres para a pesca, elas seguem invisíveis nas estatísticas pesqueiras, seja no Brasil ou em Gâmbia, Vietnam, Nigéria, Tanzânia, Tailândia, Camboja, Ilhas do Pacífico, África do Sul, Finlândia, Inglaterra ou México (HARPER et al., 2013; LENTISCO; LEE, 2015; SALMI; SONCK-RAUTIO, 2018; ZHAO et al., 2013). Tal invisibilidade feminina nas estatísticas pesqueiras é um reflexo da invisibilidade do papel da mulher no setor como um todo, proporcionada tanto por homens quanto por mulheres e sustentada em valores culturais que tendem a 
subvalorizar o trabalho feminino e supervalorizar o masculino (GUSTAVSSON; RILEY, 2018; KORALAGAMA; GUPTA; POUW, 2017; YODANIS, 2000).

A invisibilidade do papel da mulher na pesca também se relaciona ao fato de estarem frequentemente mais associadas à pesca de subsistência, a qual tem sido negligenciada em grande parte das estatísticas pesqueiras oficiais (HARPER et al., 2013). No entanto, a pesca de subsistência, incluindo a cata de mariscos, possui importância crucial na redução da pobreza, na soberania alimentar e na garantia da segurança alimentar, principalmente em países menos desenvolvidos e emergentes (KÉBÉ, 2009). A atividade pesqueira das mulheres muitas vezes é responsável por uma porção considerável da dieta diária de suas famílias, representando uma fonte essencial de proteínas e micronutrientes, raramente considerada (HARPER et al., 2013).

Além das várias atuações diretas nas atividades pesqueiras, é importante atentar também para os tantos aportes indiretos que as mulheres geram para o setor pesqueiro. Por exemplo, ao assumir diversas demandas da família (e.g. criação dos filhos, provimento da alimentação básica, manutenção da casa), as mulheres muitas vezes acabam subsidiando as atividades de pesca de seus maridos (COFFEY et al., 2020). Estes investimentos de "bastidores" não tendem a entrar na conta dos rendimentos da pesca. No entanto, tais papéis femininos também possuem um grande impacto econômico, pois subsidiam a pesca comercial e contribuem para a estabilidade social das famílias (HARPER et al., 2013).

Quadro 2-O papel feminino na pesca ao redor do mundo.

As atividades das mulheres na pesca variam ao redor do mundo. Por exemplo, considerando as atividades de captura e pós-captura, as mulheres representam $75 \%$ da força de trabalho na Nigéria, $72 \%$ na Índia, $57 \%$ no Camboja, $40 \%$ em Gana, $33 \%$ na Islândia, 32\% no Senegal, 30\% no Brasil, 21\% na União Europeia e 19\% na China (MONFORT, 2015). Globalmente, as mulheres respondem por $47 \%$ da força de trabalho nos setores de pesca (captura e pós-captura), correspondendo a 56 milhões de mulheres engajadas nesta atividade (WORLD BANK; FAO; WORLDFISH CENTER, 2012). Em geral, o setor de póscaptura é o que detém a maior concentração de atuação feminina, mas as mulheres chegam a responder por 14\% (8,3 milhões) dos trabalhadores engajados no setor de captura (FAO, 2018). Em algumas regiões, 25-55\% de toda a captura da pesca artesanal de pequena escala é garantida por mulheres (HARPER et al., 2013; KLEIBER; HARRIS; VINCENT, 2014b; LENTISCO; LEE, 2015). Em muitos países, as mulheres vão pescar em pequenos barcos em lagos e rios, e muitas em embarcações próprias, como no Benin, no Camboja, Congo, Mali e Tailândia (MONFORT, 2015). Ainda assim, não é incomum que mulheres tenham que lidar com tabus culturais, como aqueles que atribuem má sorte na pesca caso as mesmas estejam na embarcação. De forma geral, no entanto, e independente de sua maior ou menor participação no setor pesqueiro de um país, prevalece a exclusão da mulher no planejamento e na formulação de políticas públicas (DI CIOMMO; SCHIAVETTI, 2012).

\section{A mulher e a distribuição da renda da pesca}

A invisibilidade da força de trabalho feminina no setor pesqueiro frequentemente resulta na inadequada ou não remuneração da mulher, uma vez que a participação feminina é muitas vezes tida como uma ajuda informal ou uma extensão das obrigações domésticas (DI CIOMMO; SCHIAVETTI, 2012; YODANIS, 2000). Considerando que o valor socialmente atribuído a determinado trabalho tende a estar relacionado à remuneração do mesmo (COFFEY et al., 2020), a falta de reconhecimento financeiro da força de trabalho feminina pode retroalimentar a invisibilidade do papel feminino, tornando-se ao mesmo tempo causa e consequência de tal invisibilidade. Neste sentido, garantir a adequada remuneração das mulheres pelas atividades prestadas vai muito além de prover um dinheiro que lhes é merecido e devido. É também uma maneira de quebrar as barreiras da invisibilidade e de tornar mais evidente, tanto para elas quanto para seus pares, a importância de seu papel socioeconômico (ZHAO et al., 2013).

Além disso, garantir que o lucro proveniente da pesca seja dividido entre todos os que participam da atividade, ao invés de direcionado apenas ao homem como "representante" da família, poderia abrir muitas janelas de oportunidade. Primeiramente, poderia prover renda diretamente para a mulher, contribuindo para que ela ganhe mais autonomia e poder (ABRAMS, 2012). Ao ter o próprio dinheiro em mãos, as mulheres são liberadas da necessidade de ter que pedir aos maridos a quantia necessária para comprar qualquer item que desejem. Isto reduz a dependência e a vulnerabilidade femininas, e isenta as mulheres da necessidade de subjugar todas as suas vontades ao julgamento e aprovação do homem (FREITAS et al., 2020).

Em segundo lugar, a distribuição da renda entre homem e mulher dentro da unidade doméstica tende a promover diversos benefícios para a família como um todo. Estudos em diferentes países mostram que a renda sob controle feminino normalmente gera mais benefícios para os filhos (LUNDBERG; POLLAK; WALES, 1997; THOMAS, 1990; WARD-BATTS, 2008) e maior 
investimento familiar em itens alimentícios, melhorando o status nutricional dos membros familiares e de sua saúde (DOSS, 2006; HODDINOTT; HADDAD, 1995; THOMAS, 1990). Um estudo conduzido no Brasil, por exemplo, mostrou que a probabilidade de sobrevivência infantil é quase 20 vezes maior quando a renda é controlada pela mãe do que quando controlada pelo pai (THOMAS, 1990). No Reino Unido, a transferência da renda do homem para a mulher resultou na diminuição das despesas familiares com álcool e roupas masculinas, e no aumento do investimento em brinquedos e roupas femininas e infantis (LUNDBERG; POLLAK; WALES, 1997; WARD-BATTS, 2008). Na Costa do Marfim, um aumento do domínio feminino sobre a renda gerou uma diminuição dos gastos familiares com álcool e cigarros (HODDINOTT; HADDAD, 1995). Esses estudos tornam evidente que a unidade familiar não deve ser vista como um ator econômico único, uma vez que existem distintas tendências de alocação de recursos, de acordo com as preferências individuais e prioridades de homens e mulheres (DOSS, 2006). Portanto, garantir que as mulheres sejam diretamente remuneradas pela sua participação nas atividades relacionadas à pesca significa não apenas promover um cenário mais justo no setor pesqueiro, mas também uma vida provavelmente mais saudável e digna para elas e suas famílias.

\section{A mulher, a gestão pesqueira e os direitos trabalhistas}

Para entender o papel feminino na gestão pesqueira é importante voltar um pouco à história das atividades pesqueiras e dos movimentos sociais no Brasil, já que a forte intervenção inicial do Estado na pesca pode explicar em parte esse cenário (DIEGUES, 2004).

A pesca no Brasil Colonial incluía três atividades: a primeira feita pelos escravos para seu próprio consumo, após o seu trabalho (forçado) regular; a segunda, feita pelos escravos que participavam do comércio de pescado através de seus senhores; e a terceira, feita pelos pescadores livres, a maioria indígena, que vendiam o peixe nos mercados (SILVA, 1988: 45). O controle do Estado no Brasil, no século XIX, incluía inclusive o dízimo do pescado, cobrado pelas câmaras municipais. Além disso, os locais permitidos para a venda do pescado eram determinados pelas posturas municipais (SILVA, 1988: 117, 121). A primeira regulamentação de pescadores ocorreu em 1846, através das Capitanias dos Portos nas províncias marítimas do Império (SILVA, 1988: 124). Este primeiro registro de pescadores nas Capitanias dos Portos teve sentido militar e de controle marítimo (SILVA, 1988: 135), o que nos leva a destacar que, ao relacionar a pesca a uma atividade militar, a exclusão das mulheres já seria esperada.

Em 1912, o governo criou as Colônias de Pescadores, que visavam garantir um grupo de reserva para a defesa do país (novamente uma oportunidade de militarização da pesca, em função dos conhecimentos marinhos úteis em caso de guerra) e para servir como centros educacionais de integração do pescador com o país (BRETON et al., 1996). Entre 1932 e 1961, as colônias estiveram associadas ou ao Ministério da Marinha ou ao Ministério da Agricultura. No governo militar, elas eram comandadas por sargentos ou tenentes (BRETON et al., 1996), o que evidencia que as colônias nascem e se mantêm por longo prazo sem representatividade social.

Apenas com a queda da ditadura no Brasil, em 1985, associações e colônias pesqueiras livres, espontâneas, e sem o "incentivo" militar começaram a ser formadas na costa brasileira. No Rio de Janeiro, há algumas que incluem mulheres em sua direção, como a Colônia de Pescadores de Copacabana (Z13) e a de Arraial do Cabo (Z5) (A.B., obs. pes.). No Rio Grande do Norte, ao menos oito colônias das 19 do Estado estavam sob controle feminino em 2020 (P.F.M.L., obs. pes.), algo que se repete em vários outros Estados brasileiros. De fato, a partir dos anos 1990 as mulheres passam a se mobilizar nacionalmente para influenciar ações do Estado que as contemplassem como pescadoras, incluindo o questionamento das dificuldades de acesso às políticas sociais e previdenciárias e ao poder político dentro do setor pesqueiro, como a possibilidade de liderarem organizações de pesca (colônias, sindicatos e associações) (ALENCAR; PALHETA; SOUSA, 2015). Em 2009, os caminhos para superar essas dificuldades foram facilitados, através da Lei no. 11.959 de 29 de junho de 2009, conhecida como a Lei da Pesca. Essa lei permitiu a inscrição no Registro Geral da Atividade Pesqueira a qualquer pessoa que exercesse a atividade pesqueira, a qual, por sua vez, contemplou no contexto artesanal desde a confecção e reparo de apetrechos até a comercialização do pescado. Assim, estavam abertas as portas para a maior participação oficial feminina, para além da extração de recursos. No entanto, esta lei não marca o fim de uma luta histórica pelo reconhecimento feminino nas atividades pesqueiras, já que nos anos seguintes novos decretos e medidas provisórias (e.g., Medidas Provisórias 664 e 665 e Decretos Presidenciais 8.424/2015 e 8.425/2015) reclassificaram e restringiram a definição de pesca e de atividades de "apoio à pesca" (e.g. beneficiamento do pescado, produção e manutenção de apetrechos) para impedir que os "pescadores não legítimos" (qualquer pessoa que não trabalhe na extração de peixes 
e mariscos) tivessem acesso a direitos como seguro-defeso, aposentadoria e pensão. Assim, mulheres que são classificadas como aquelas que provêm apoio à pesca não têm direito à licença maternidade, seguro desemprego, auxílio doença ou contagem do tempo para aposentadoria especial (SILVA; LEITÃO, 2012).

Muito da articulação feminina que se seguiu nos últimos anos surgiu a partir de movimentos de base, como a Articulação Nacional das Pescadoras do Brasil, fundada em 2005, após acenos do governo em reconhecer suas demandas, que não resultaram em ações concretas. Hoje, estas demandas incluem maior acesso aos direitos trabalhistas, o reconhecimento do território pesqueiro, os riscos da privatização das águas e o reconhecimento das doenças ocupacionais, que tendem a afetar às pescadoras de modo distinto que aos pescadores. A falta de reconhecimento das doenças ocupacionais decorrentes da pesca feminina é um ponto especialmente nevrálgico que limita o acesso universal aos direitos trabalhistas das pescadoras (STADTLER, 2013). Doenças ocupacionais, como lesões decorrentes do carregamento de pesados baldes de mariscos, por exposição constante à água (mariscadoras trabalham com parte do corpo constantemente molhado), por acidentes como o pisoteio de peixes venenosos (e.g., Thalassophryne spp., peixe-sapo venenoso) e/ou por contato mais direto e constante com ambientes eventualmente muito poluídos, como mangues e estuários, são dificilmente reconhecidas como tais (ROCHA et al., 2014). Parte desta dificuldade é atribuída ao machismo estrutural da sociedade, presente ainda em muitas colônias presididas por homens que se recusam a cadastrar mulheres, mas também presente no Sistema Básico de Saúde. Segundo relatos informais de pescadoras e da ONG Oceânica (Pirangi/RN) a que tivemos acesso, não é incomum atendentes de postos de saúde minimizarem as queixas de saúde femininas decorrentes da atividade pesqueira porque as mulheres comparecem ao local de unhas pintadas, por exemplo (Rocha, com. pes.). Neste caso, a pesca feminina seria encarada como um impeditivo para a autoestima: a uma pescadora estaria negado o direito de se arrumar para buscar ajuda no sistema público de saúde. Segundo os mesmos relatos, muitos atendentes também minimizam o fato de que mariscadoras carregam sacos e baldes de conchas pesando até $40 \mathrm{~kg}$, múltiplas vezes em um único dia, em corpos muitas vezes já fragilizados pela jornada dupla de cuidar da família (RAINHO, 2017).

\section{A mulher nos processos de comanejo e organização comunitária}

Nas últimas décadas, tem se destacado ao redor do mundo a implementação de sistemas de manejo colaborativo de recursos naturais, usualmente denominados "sistemas de comanejo". Essas iniciativas passaram a ocorrer mais expressivamente a partir da década de 1980, representando uma alternativa ao regime de livre acesso (open access) e à centralização do controle nas mãos do Estado (CLEAVER, 2000; GUTIÉRREZ; HILBORN; DEFEO, 2011). Em termos gerais, o comanejo refere-se ao compartilhamento do direito de tomada de decisão e de controle sobre o uso dos recursos entre os usuários e o Estado ou demais instituições envolvidas (JENTOFT, 1989; POMEROY; BERKES, 1997). Acredita-se que a partir desse sistema mais inclusivo o manejo tenda a apresentar maior eficiência, pois as regras passam a ser mais legítimas para os usuários e a refletir melhor as condições do local, resultando em propostas mais coerentes e em uma maior predisposição ao cumprimento dos acordos (JENTOFT, 2000; JENTOFT; MCCAY; WILSON, 1998; MCCLANAHAN et al., 2006, 2011).

Apesar da existência de uma vasta literatura voltada para os regimes de comanejo, abordando seus resultados e processos de implementação (CINNER et al., 2012; GUTIÉRREZ; HILBORN; DEFEO, 2011; JENTOFT, 2000; MCCLANAHAN et al., 2006), estudos com foco na participação das mulheres em tais regimes são relativamente escassos. Isso pode ser consequência, ainda que paradoxalmente, de as mulheres corresponderem a um dos grupos comumente excluídos de participação efetiva em iniciativas aparentemente participativas (AGARWAL, 2001). Tal exclusão feminina muitas vezes é resultado de percepções sociais que restringem o papel das mulheres e desmerecem as habilidades femininas (AGARWAL, 2000).

No entanto, estudos têm encontrado relações positivas entre a representação feminina em sistemas de comanejo de recursos naturais e o sucesso desses sistemas (AGARWAL, 2009; STAPLES; NATCHER, 2015; WESTERMANN; ASHBY; PRETTY, 2005). À presença da mulher foi atribuído, por exemplo, aumento dos níveis de cooperação, solidariedade, resolução de conflitos e capacidade de ação coletiva autossustentável (WESTERMANN; ASHBY; PRETTY, 2005). A participação feminina também parece trazer abordagens mais holísticas para as tomadas de decisão, contribuindo para discussões mais complexas e para o estabelecimento de uma visão de longo-prazo dos problemas de gestão (STAPLES; NATCHER, 2015). Além disso, uma maior proporção de mulheres em grupos de 
comanejo tende a garantir melhores resultados para os recursos em foco, devido principalmente a um maior nível de proteção dos recursos e de cumprimento das regras, e à maior cooperação existente entre mulheres (AGARWAL, 2009).

A participação feminina nas iniciativas de comanejo é historicamente deficiente na América Latina. Por exemplo, no começo dos anos 2000, em um livro especialmente organizado sobre o comanejo em comunidades pesqueiras na região (WILSON; NIELSEN; DEGNBOL, 2003), o termo gender aparece apenas uma vez no índice remissivo e o termo woman/women, nenhuma. De fato, a única vez em que o termo gender aparece é para explicar como a pesca e seu manejo têm viés fortemente masculino, com a exceção, à época, do caso de Pearl Lagoon, na Nicarágua (BEGOSSI; BROWN, 2003). No entanto, já havia evidências de que novos ventos sopravam por aqui, como a inserção feminina em representações importantes, a exemplo da CFRAMP (Caribbean Community Fisheries Resource Assessment and Management Programme) (BEGOSSI; BROWN, 2003).

Um outro bom exemplo destes novos tempos é a inclusão feminina no comanejo do pirarucu, na Amazônia (FREITAS et al., 2020). O pirarucu (Arapaima spp.) é um dos maiores peixes de escama de água doce do mundo e foi o principal recurso pesqueiro comercial da Amazônia brasileira durante os séculos XIX e XX (MÉRONA, 1993; VERÍSSIMO, 1895). Os níveis intensos de exploração levaram as populações da espécie a quadros de depleção e até extinções locais, o que fez com que sua pesca se tornasse proibida no final dos anos 1990 (CASTELLO et al., 2015; CASTELLO; STEWART, 2010). No entanto, a pesca ilegal do pirarucu se manteve muito presente em toda a Amazônia, fazendo com que as populações continuassem declinando (CAVOLE; ARANTES; CASTELLO, 2015). Esse cenário mudou apenas após o surgimento de um modelo de comanejo de pirarucu, na Reserva de Desenvolvimento Sustentável Mamirauá, o qual demonstrou grande sucesso ecológico e social, passando a ser replicado em diversas outras áreas da Amazônia (CAMPOS-SILVA; PERES, 2016; CASTELLO et al., 2009; PETERSEN et al., 2016). Tal modelo de comanejo se baseia em uma série de regras, exigidas pelo Instituto Brasileiro do Meio Ambiente e dos Recursos Naturais Renováveis (IBAMA; Decreto no. 36.083, 23 de julho de 2015, Amazonas). Apesar de historicamente a pesca do pirarucu ser tida como uma atividade muito especializada e restrita a poucos "especialistas" locais (sempre homens), o estabelecimento desse modelo de comanejo fez com que a atividade adquirisse um perfil coletivo (CASTELLO et al., 2009). Neste cenário, algumas comunidades passaram a incluir não apenas os "não-especialistas" homens na cadeia de produção, como também muitas mulheres (FREITAS et al., 2020). Tal inclusão foi possibilitada por algumas características advindas desse novo arranjo do regime de comanejo, como o sistema de divisão de tarefas e as oportunidades de capacitação dos membros comunitários para atuação nas etapas de captura e pós-captura. As mulheres vêm sendo remuneradas pela sua participação, o que representa uma quebra de paradigma na região, uma vez que a grande maioria delas nunca havia sido remunerada por seu desempenho em atividades pesqueiras, apesar de muitas frequentemente participarem de pescarias comerciais. A despeito de tais avanços positivos, rumo a uma maior equidade de gênero, é preciso ressaltar que a inclusão feminina no comanejo do pirarucu ainda não é uma realidade comum a todas as áreas que vêm desenvolvendo esse manejo. Mesmo naquelas em que as mulheres já vêm participando, muitos avanços ainda são necessários para que elas sejam mais ativas nas tomadas de decisão, de forma equiparada aos homens (FREITAS $e t$ al., 2020).

\section{CONCLUSÃO}

Nas últimas décadas, pescadoras e mariscadoras ao redor do mundo e no Brasil vêm ganhando algum reconhecimento e começam, mesmo que timidamente, a ocupar postos de liderança ou a ser devidamente remuneradas por sua atividade. Por outro lado, vencer de vez a invisibilidade feminina no setor pesqueiro, especialmente o brasileiro, requer contestar um modelo historicamente enviesado em favor dos homens, desde o surgimento das primeiras regulamentações aplicadas à pesca. $\mathrm{O}$ fim da invisibilidade das pescadoras demandará esforços em diferentes frentes, e cabe um forte apelo à academia para que promova cada vez mais este debate e à sociedade para que o dissemine. Será preciso demonstrar e reforçar, por estudos e depois por ações, os pontos que trouxemos aqui: 1) mulheres pescam e exercem papel crucial na segurança alimentar de suas famílias e comunidades, mas suas capturas são raramente computadas; 2) em alguns casos pescadoras assumem postos de liderança, mas em muitos outros ainda são excluídas da oportunidade de se afiliarem em colônias, a depender do entendimento do presidente em exercício sobre o que constitui um(a) pescador(a); 3) pescadoras podem e devem ter melhor retorno financeiro da atividade pesqueira, até porque por vezes fazem melhor uso 
familiar deste dinheiro que seus maridos; e 4) pescadoras necessitam ter seus direitos trabalhistas reconhecidos, o que inclui ter suas doenças laborais legitimadas e respeitadas pelo sistema de saúde. Ao se ignorar o papel das pescadoras na sociedade, reforçamos a falta de reconhecimento da mulher, seja ela quem for e qual profissão exerça, como sujeito ativo, político, econômico e detentor de direitos.

\section{AGRADECIMENTOS}

A todas as pescadoras, marisqueiras e mulheres do mar e dos rios que ao longo de muitos anos abrem as portas de suas casas para nós, com um sorriso estampado no rosto, apesar de todas as agruras do cotidiano das comunidades pesqueiras. À Lígia Rocha (ONG Oceânica) que contribui ao nos lembrar que a invisibilidade não é só nas estatísticas, mas é nos postos de saúde, no reconhecimento das doenças da mariscagem e na dificuldade em acessarem seus direitos trabalhistas como pescadores. AB e PFML agradecem ao CNPq por suas bolsas de produtividade (301592/2017-9 e 301515/2019-0, respectivamente). CTF e PFML agradecem à Fundação de Amparo à Pesquisa do Estado de São Paulo (FAPESP) por sua bolsa de pós-doutorado (processo $n^{\circ}$ 2019/15550-2) e por sua bolsa de pesquisador visitante (2018/22087-4), respectivamente.

\section{REFERÊNCIAS}

ABRAMS, L. "There Is Many a Thing That Can Be Done with Money": Women, Barter, and Autonomy in a Scottish Fishing Community in the Nineteenth and Twentieth Centuries. Signs: Journal of Women in Culture and Society, v. 37, n. 3, p. 602-609, 1 mar. 2012.

AGARWAL, B. Conceptualising environmental collective action: why gender matters. Cambridge Journal of Economics, v. 24, n. 3, p. 283-310, 2000.

AGARWAL, B. Participatory Exclusions, Community Forestry, and Gender: An Analysis for South Asia and a Conceptual Framework. World Development, v. 29, n. 10, p. 1623-1648, 2001.

AGARWAL, B. Gender and forest conservation: The impact of women's participation in community forest governance. Ecological Economics, v. 68, n. 11, p. 2785-2799, 2009.

ALENCAR, E. F.; PALHETA, S. P.; SOUSA, I. S. Trabalho na pesca, ação política e identidade: as mulheres da Colônia de Pescadores Z-32 de Maraã-Amazonas. In: SCHERER, E. F. (Ed.). . Aqui estamos: entre as águas dos mares, águas dos rios, nas terras de trabalho da pesca artesanal. Rio de Janeiro: Letra Capital Editora, 2015. p. 39-70.

ARCE-IBARRA, A. M.; CHARLES, A. T. Inland fisheries of the Mayan Zone in Quintana Roo, Mexico: Using a combined approach to fishery assessment for data-sparse fisheries. Fisheries Research, v. 91, n. 2, p. 151-159, 2008.

BEGOSSI, A.; BROWN, D. Experiences with fisheries co-management in Latin America and the Caribbean. In: WILSON, D. C.; NIELSEN, J. R.; DEGNBOL, P. (Eds.). . The fisheries co-management experience. Fish and Fisheries Series. Dordrecht: Springer, 2003. v. 26, p. 135-152.

BÉNÉ, C.; MACFADYEN, G.; ALLISON, E. H. Increasing the contribution of small-scale fisheries to poverty alleviation and food security. [s.l.] Food and Agriculture Organization of the United Nations, 2007.

BÉNÉ, C.; TEWFIK, A. Fishing Effort Allocation and Fishermen's Decision Making Process in a MultiSpecies Small-Scale Fishery: Analysis of the Conch and Lobster Fishery in Turks and Caicos Islands. Human Ecology, v. 29, n. 2, p. 157-186, 2001.

BRANCH, T. A.; KLEIBER, D. Should we call them fishers or fishermen? Fish and Fisheries, v. 18, n. 1, p. 114-127, 2017.

BRETON, Y. et al. Fisheries management and the Colonias in Brazil: A case study of a top-down producers' organization. Society \& Natural Resources, v. 9, n. 3, p. 307-315, 1996.

BRUMMETT, R. E. et al. Women's traditional fishery and alternative aquatic resource livelihood strategies in the Southern Cameroonian Rainforest. Fisheries Management and Ecology, v. 17, n. 3, p. 221-230, 2010.

CALHOUN, S.; CONWAY, F.; RUSSELL, S. Acknowledging the voice of women: implications for fisheries management and policy. Marine Policy, v. 74, p. 292-299, 2016.

CAMPOS-SILVA, J. V.; PERES, C. A. Community-based management induces rapid recovery of a high-value tropical freshwater fishery. Scientific Reports, v. 6, p. 34745, 2016.

CASTELLO, L. et al. Lessons from integrating fishers of arapaima in small-scale fisheries management at the Mamirauá Reserve, Amazon. Environmental Management, v. 43, p. 197-209, 2009.

CASTELLO, L. et al. Understanding fishing-induced extinctions in the Amazon. Aquatic Conservation: Marine and Freshwater Ecosystems, v. 25, n. 5, p. 587-598, 2015.

CASTELLO, L.; STEWART, D. J. Assessing CITES non-detriment findings procedures for Arapaima in Brazil. Journal of Applied Ichthyology, v. 26, n. 1, p. 49-56, 2010.

CAVOLE, L. M.; ARANTES, C. C.; CASTELLO, L. How illegal are tropical small-scale fisheries? An estimate for arapaima in the Amazon. Fisheries Research, v. 168, p. 1-5, 2015. 
CHRISTENSEN, J. B. Motor power and women power: Technological and economic change among the Fanti fishermen of Ghana. In: SMITH, E. (Ed.). . Those who live from the sea: a study in maritime anthropology. Saint Paul: West Pub. Co., 1977. p. 71-96.

CINNER, J. E. et al. Comanagement of coral reef social-ecological systems. Proceedings of the National Academy of Sciences, v. 109, n. 14, p. 5219-5222, 2012.

CLEAVER, F. Analysing gender roles in community natural resource management: negotiation, lifecourses and social inclusion. IDS Bulletin, v. 31, n. 2, p. 60-67, 2000.

COFFEY, C. et al. Time to care: unpaid and underpaid care work and the global inequality crisis. Oxford: Oxfam, 2020. Disponível em: <https://oxfamilibrary.openrepository.com/bitstream/handle/10546/620928/bptime-to-care-inequality-200120-en.pdf $>$.

DAMÁSIO, L. M. A.; PENINNO, M. G.; LOPES, P. F. M. Small changes, big impacts: Geographic expansion in small-scale fisheries. Fisheries Research, v. 226, p. 105533, 2020.

DE OLIVEIRA LEIS, M. et al. Overview of Small-Scale Fisheries in Latin America and the Caribbean: Challenges and Prospects. In: SALAS, S.; BARRAGÁN-PALADINES, M. J.; CHUENPAGDEE, R. (Eds.). . Viability and Sustainability of Small-Scale Fisheries in Latin America and The Caribbean. MARE Publication Series. Cham: Springer International Publishing, 2019. p. 15-47.

DI CIOMMO, R. C.; SCHIAVETTI, A. Women participation in the management of a Marine Protected Area in Brazil. Ocean \& Coastal Management, v. 62, p. 15-23, 2012.

DIEGUES, A. C. S. O olhar estrangeiro. São Paulo: Hucitec/Nupaub/CEC/USP, 2004. v. 3

DOSS, C. The Effects of Intrahousehold Property Ownership on Expenditure Patterns in Ghana. Journal of African Economies, v. 15, n. 1, p. 149-180, 2006.

FAO. The State of World Fisheries and Aquaculture 2018: Meeting the sustainable development goals. Rome, Italy: FAO, 2018. 1966.

FIRTH, R. The Malay fishermen: their peasant economy. New York: The W.W. Norton \& Company, Inc.,

FREITAS, C. T. et al. Co-management: A Potential Tool to Promote Gender Equity in Fisheries. Ecological Economics, v. 176, p. 106709, 2020.

FRÖCKLIN, S. et al. Fish Traders as Key Actors in Fisheries: Gender and Adaptive Management. AMBIO, v. 42, n. 8, p. 951-962, 2013.

GUSTAVSSON, M.; RILEY, M. Women, capitals and fishing lives: exploring gendered dynamics in the Llŷn Peninsula small-scale fishery (Wales, UK). Maritime Studies, v. 17, n. 2, p. 223-231, 2018.

GUTIÉRREZ, N. L.; HILBORN, R.; DEFEO, O. Leadership, social capital and incentives promote successful fisheries. Nature, v. 2, p. 5-8, 2011.

HARPER, S. et al. Women and fisheries: Contribution to food security and local economies. Marine Policy, V. 39, p. 56-63, 2013.

HARPER, S. et al. Valuing invisible catches: Estimating the global contribution by women to small-scale marine capture fisheries production. PLOS ONE, v. 15, n. 3, p. e0228912, 2020.

HAWKES, K.; BIRD, R. B. Showing off, handicap signaling, and the evolution of men's work. Evolutionary Anthropology: Issues, News, and Reviews, v. 11, n. 2, p. 58-67, 2002.

HODDINOTT, J.; HADDAD, L. Does Female Income Share Influence Household Expenditures? Evidence from Côte D'ivoire. Oxford Bulletin of Economics and Statistics, v. 57, n. 1, p. 77-96, 1995.

JENTOFT, S. Fisheries co-management: Delegating government responsibility to fishermen's organizations.

Marine Policy, v. 13, n. 2, p. 137-154, 1 abr. 1989.

JENTOFT, S. Legitimacy and disappointment in fisheries management. Marine Policy, v. 24, n. 2, p. 141148,2000

JENTOFT, S. Introduction. In: KOOIMAN, J. et al. (Eds.). . Fish for life: Interactive governance for fisheries. MARE Publication Series. Amsterdam: Amsterdam University Press, 2005. p. 147-152.

JENTOFT, S.; MCCAY, B. J.; WILSON, D. C. Social theory and fisheries co-management. Marine Policy, v. 22, n. 4-5, p. 423-436, 1998.

JEYANTHI, P. et al. Employment Status of Women in the Seafood Processing Sector of Gujarat. Fishery Technology, v. 52, p. 135-139, 2015.

KÉBÉ, M. Taking the contribution of fisheries into account in development policy. In: ACKEFORS, H. (Ed.). Fisheries, sustainability and development: fifty- two authors on coexistence and development of fisheries and aquaculture in developing countries. Stockholm: Royal Swedish Academy of Agriculture and Forestry (KSLA), 2009. p. 365-375.

KERGOAT, D. Divisnao sexual do trabalho e relações sociais de sexo. In: HIRATA, H. et al. (Eds.). Dicionário crítico do feminismo. São Paulo: Editora Unesp, 2009. p. 67-76.

KLEIBER, D.; HARRIS, L. M.; VINCENT, A. C. J. Gender and small-scale fisheries: a case for counting women and beyond. Fish and Fisheries, v. 16, n. 4, p. 547-562, 2014a.

KLEIBER, D.; HARRIS, L. M.; VINCENT, A. C. J. Improving fisheries estimates by including women's catch in the Central Philippines. Canadian Journal of Fisheries and Aquatic Sciences, v. 71, n. 5, p. 656-664, 2014 b. 
KORALAGAMA, D.; GUPTA, J.; POUW, N. Inclusive development from a gender perspective in small scale fisheries. Current Opinion in Environmental Sustainability, Sustainability science. v. 24, p. 1-6, 2017.

LENTISCO, A.; LEE, R. U. A review of women's access to fish in small-scale fisheries. FAO Fisheries and Aquaculture Circular, v. 1098, p. 1-36, 2015.

LUNDBERG, S. J.; POLLAK, R. A.; WALES, T. J. Do Husbands and Wives Pool Their Resources? Evidence from the United Kingdom Child Benefit. The Journal of Human Resources, v. 32, n. 3, p. 463-480, 1997.

MAGALHÃES, A. et al. The role of women in the mangrove crab (Ucides cordatus, Ocypodidae) production process in North Brazil (Amazon region, Pará). Ecological Economics, v. 61, n. 2, p. 559-565, 2007.

MCCLANAHAN, T. R. et al. A comparison of marine protected areas and alternative approaches to coral-reef management. Current Biology, v. 16, p. 1408-1413, 2006.

MCCLANAHAN, T. R. et al. Critical thresholds and tangible targets for ecosystem-based management of coral reef fisheries. Proceedings of the National Academy of Sciences, v. 108, n. 41, p. 17230-17233, 2011.

MÉRONA, B. DE. Pesca e ecologia dos recursos aquáticos na Amazônia. In: FURTADO, L. G.; LEITAO, W.; FIUZA DE MELLO, A. (Eds.). . Povos das aguas realidade e perspectivas na Amazonia. Coleçao Eduardo Galvao. Belem: Museu Paraense Emilio Goeldi, 1993. p. 159-185.

MONFORT, M. C. The role of women in the seafood industry. Rome: Food and Agriculture Organization, 2015. v. 119

OGDEN, L. E. Fisherwomen-The Uncounted Dimension in Fisheries ManagementShedding light on the invisible gender. BioScience, v. 67, n. 2, p. 111-117, 2017.

PETERSEN, T. A. et al. Recovery of Arapaima sp. populations by community-based management in floodplains of the Purus River, Amazon. Journal of Fish Biology, v. 89, n. 1, p. 241-248, 2016.

POMEROY, R. S.; BERKES, F. Two to tango: The role of government in fisheries co-management. Marine

Policy, v. 21, n. 5, p. 465-480, 1997.

RAINHO, A. P. A backbreaking struggle: SAMUDRA Report. Chennai, India: International Collective in Support of Fish Workers, 2017.

RETIRED, M. P.; MBEZI, R. G. From Hand to Mouth: Fishery Projects, Women, Men and Household Poverty. Canadian Journal of Development Studies / Revue canadienne d'études du développement, v. 31, n. 3-4, p. 381-400, 2010.

ROCHA, L. M. et al. Biologia, ecologia, cultivo e aspectos sócio-econômicos da mariscagem do molusco Anomalocardia brasiliana no Brasil. In: Gente da maré: aspectos ecológicos e socieconômicos da mariscagem no Nordeste Brasileiro. Mossoró: Edufersa, 2014. p. 157-184.

ROCHA, L. M.; PINKERTON, E. Comanagement of clams in Brazil: a framework to advance comparison. Ecology and Society, v. 20, n. 1, 2015.

SALMI, P.; SONCK-RAUTIO, K. Invisible work, ignored knowledge? Changing gender roles, division of labor, and household strategies in Finnish small-scale fisheries. Maritime Studies, v. 17, n. 2, p. 213-221, 2018. SILVA, L. G. Os pescadores na História do Brasil: colônia e império. Recife: Comissão Pastoral dos Pescadores, 1988.

SILVA, V. L.; LEITÃO, M. DO R. DE F. A. A regulação jurídica da pesca artesanal no Brasil e o problema do reconhecimento do trabalho profissional das pescadoras. In: $17^{\mathrm{O}}$ ENCONTRO NACIONAL DA REDE FEMINISTA NORTE E NORDESTE DE ESTUDOS E PESQUISAS SOBRE A MULHER E RELAÇÕES DE GÊNERO - REDOR. João Pessoa: 2012

STADTLER, H. Mulheres na pesca artesanal: lutrando por previdência e saúde. In: SEMINÁRIO INTERNACIONAL FAZENDO GÊNERO 10: DESAFIOS ATUAIS DO FEMINISMO. Florianópolis: 2013 Disponível

em: <http://www.fg2013.wwc2017.eventos.dype.com.br/resources/anais/20/1384892758_ARQUIVO_HuldaStadtler. pdf $>$

STAPLES, K.; NATCHER, D. C. Gender, Decision Making, and Natural Resource Co-management in Yukon. Arctic, v. 68, n. 3, p. 356-366, 2015.

SZYMKOWIAK, M. Genderizing fisheries: Assessing over thirty years of women's participation in Alaska fisheries. Marine Policy, v. 115, p. 103846, 1 maio 2020

THE WORLD BANK; FAO; WORLDFISH CENTER. The hidden harvests: the global contribution of capture fisheries. Washington, DC: The International Bank for Reconstruction and Development/ The World Bank, 2012.

THOMAS, D. Intra-Household Resource Allocation: An Inferential Approach. The Journal of Human Resources, v. 25, n. 4, p. 635-664, 1990.

THOMPSON, P. Women in the Fishing: The Roots of Power between the Sexes. Comparative Studies in Society and History, v. 27, n. 1, p. 3-32, jan. 1985

VERÍSSIMO, J. A pesca na Amazônia. Rio de Janeiro: Livraria Clássica Alves \& Companhia, 1895.

WARD-BATTS, J. Out of the Wallet and into the Purse Using Micro Data to Test Income Pooling. Journal of Human Resources, v. 43, n. 2, p. 325-351, 2008. 
WEERATUNGE, N.; SNYDER, K. A.; SZE, C. P. Gleaner, fisher, trader, processor: understanding gendered employment in fisheries and aquaculture. Fish and Fisheries, v. 11, n. 4, p. 405-420, 2010.

WESTERMANN, O.; ASHBY, J.; PRETTY, J. Gender and social capital: The importance of gender differences for the maturity and effectiveness of natural resource management groups. World Development, v. 33, n. 11, p. 1783-1799, 2005.

WILSON, D. C.; NIELSEN, J. R.; DEGNBOL, P. (EDS.). The fisheries co-management experience: accomplishments, challenges and prospects. Dodrecht: Springer, 2003. v. 26

YODANIS, C. L. Constructing Gender and Occupational Segregation: A Study of Women and Work in Fishing Communities. Qualitative Sociology, v. 23, n. 3, p. 267-290, 2000.

ZHAO, M. et al. Women as visible and invisible workers in fisheries: A case study of Northern England. Marine Policy, Social and cultural impacts of marine fisheries. v. 37, p. 69-76, 2013. 